\title{
Quercetin as a Modulator of Diabetic Macrovascular Complications in Murine and Chick Embryo Models
}

\author{
Joyani Das, Papiya Mitra Mazumder* \\ Department of Pharmaceutical Sciences and Technology, Birla Institute of Technology, Mesra, Ranchi-835215, Jharkand, INDIA.
}

\begin{abstract}
Background: Quercetin, a bioflavonoid, with wide natural occurrence has been found to possess numerous pharmacological benefits. Aim: The present work deals with exploring efficacy of Quercetin against diabetic macrovascular complications taking the aid of the high fat diet fed-low dose streptozotocin rat model. The use of the chick embryo model in this regard was attempted with an aim to address the growing ethical concerns for the use of higher animals in biological experiments. Methods: High Fat Diet (HFD) + STZ $(35 \mathrm{mg} / \mathrm{kg}$ ) induced diabetes was treated with metformin and quercetin in rats. Diabetic simulation was done in chick embryos with ß-hydroxy butyric acid (15 mM) and glucose $(20 \mathrm{mM})$. HbA1c, Fasting Blood Glucose (FBG), TC, TG, HDL, LDH and CK-MB were estimated in rat serum whereas blood glucose and lipid profile tests were done in amniotic fluid, liver and heart tissues of chick embryos. Histopathological study was performed on heart tissues of the wistar albino rats. Results: Increased $\mathrm{HbA} 1 \mathrm{c}$, blood glucose, TC, TG and decreased HDL were found in the chick embryo models proving its success in simulating diabetic condition. Reversal of blood glucose, HbA1c and lipid profile anomalies towards normal after quercetin treatment was comparable to metformin treated groups in both rat and chick embryo models. Quercetin was found to be effective in decreasing elevated serum LDH and more effective in decreasing CKMB levels. Conclusion: Biochemical and histopathological evidences pointed towards quercetin having potential benefits in diabetic macrovascular complications and that the chick embryo is an effective alternative model for the said condition.
\end{abstract}

Key words: In ovo, Diabetic macrovascular complications, Chick embryo, Quercetin.

\section{INTRODUCTION}

Diabetes mellitus (DM) is a progressive metabolic disorder characterized by abnormalities in carbohydrate, fat, electrolyte and protein metabolism which ultimately lead to several acute and chronic complications. ${ }^{1}$ It has emerged as a major epidemic of this century, which has increased in incidence by $50 \%$ over the past 10 years. ${ }^{2}$ Generally, the injurious effects of hyperglycemia are separated into macrovascular complications (coronary artery disease, peripheral arterial disease, and stroke) and micro vascular complications (diabetic nephropathy, neuropathy, and retinopathy). ${ }^{3}$ The present study focusses on various macrovascular complications of DM. In diabetic patients, a much more widespread and aggressive form of atherosclerosis is observed in the coronary arteries, lower extremities, and extra cranial carotid arteries, causing nearly $80 \%$ of all deaths and much of the disability in these patients. ${ }^{4}$ The direct and indirect effects of diabetes mellitus on the human vascular tree are the major source of morbidity and mortality in both type 1 (T1D) and type 2 (T2D) diabetes where both are independent risk factors for myocardial infarction, peripheral vascular disease, and stroke with an increased risk of cardiac dysfunction and heart failure. ${ }^{5}$ In addition to atheroma formation, increased platelet adhesion and hypercoagulability occur in T2D. In diabetic patients, impaired nitric oxide generation and increased free radical formation in
Submission Date: 23-01-2018; Revision Date: 09-03-2018; Accepted Date: 17-05-2018

DOI: 10.5530/ijper.52.4.69 Correspondence: Dr. (Mrs.) Papiya Mitra Mazumder,

Department of Pharmaceutical Sciences and Technology, Birla Institute of Technology, Mesra, Ranchi, Jharkhand, INDIA.

Phone: +91 9431327044

E-mail: pmitramazumder@ bitmesra.ac.in

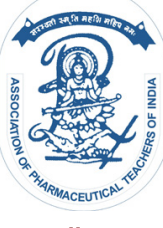

www.ijper.org 
platelets, as well as altered calcium regulation, may promote platelet aggregation. ${ }^{6}$ Elevated levels of plasminogen activator inhibitor type 1 may also impair fibrinolysis in patients with diabetes. The combination of increased coagulability and impaired fibrinolysis is likely to further increase the risk of vascular occlusion and cardiovascular events in type 2 diabetes.

Diabetic complications have been modeled in vivo and in vitro for a long time. ${ }^{7-9}$ Majority of these models deal with mammals such as mice and rats which also include genetically engineered species and cell/tissue culture specimens. ${ }^{10}$ These generally involve huge expenditure, technical support and highly trained personals. Recently there is an increased attempt to bring down the number of mammals used in biological research based on ethical grounds. ${ }^{11}$ The use of chick embryos in this respect has been proved to be fruitful. ${ }^{12}$ In this regard a model may be developed in ovo to study diabetes and also its macrovascular complications and to correlate the same to some existing murine models. The effects of protective agents may also be perceived using such a model. This will result in a non-transgenic, cost effective and relatively low duration yet precise model with greater ethical acceptance.

Flavonoids present in foods possess various medicinal properties such as antioxidant, antithrombotic, antiinflammatory, anti atherogenic, antiatherosclerotic and cardioprotective effects. Various studies have shown that the risk of heart diseases can be reduced through the consumption of flavonoid rich diets. ${ }^{13}$ They may inhibit the development of vascular diseases by altering production of endothelial cell eicosanoid. Flavonoids have shown to lower blood pressure, regulate fatty acid oxidation and improve functioning of adipocytes and are hence beneficial in obesity. ${ }^{14}$ The flavonoid quercetin has been found to be an able representative compound in this regard and its properties ought to be explored in order to evaluate the effectiveness of this flavonoid in case of diabetes induced macrovascular complications. ${ }^{15}$

\section{METHODS}

\section{In ovo model ${ }^{16}$}

Fertilized eggs (within 24 hrs of laying) of Chattisgarh Broiler (Chebro) hens were incubated at $36^{\circ}$ to $38^{\circ} \mathrm{C}$ and $58-60 \%$ R.H.

Fertilized Eggs were divided into 4 groups of 10 eggs each and were administered the following treatments on Embryonic day (ED) 10 via injection in the narrow end of eggs under aseptic conditions.

Group G1: Normal Control-Injected with $0.1 \mathrm{~mL}$ PBS, $\mathrm{pH} 7.4$
Group G2: Diabetic Control- Injected with glucose (glu 20mM) + B-hydroxy butyric acid (HBA 15mM) in $0.1 \mathrm{~mL}$ PBS (Glu+HBA).

Group G3: Standard Treated- (Glu+HBA) + Metformin (2.5mg/egg)

Group G4: Quercetin Treated- (Glu+HBA) + Quercetin (2.5mg/egg)

\section{Injection of drugs into eggs}

Each egg was candled on ED 5 and those found to be unfertilized were discarded. The process of injection was carried out on ED 10 under aseptic conditions using a bio safety cabinet equipped with ultraviolet lamp and HEPA filter. After sterilizing the surface of each egg with $70 \% \mathrm{v} / \mathrm{v}$ ethanol to minimize microbial attack to embryos, the narrow end of the egg was pierced and $0.1 \mathrm{~mL}$ drug solution as per grouping (or water for injection in case of control group eggs) was injected and the orifice was quickly sealed with molten paraffin wax. A second orifice was drilled at the blunt end to release pressure developed due to increased fluid volume and sealed immediately.

\section{Procedure after completion of 17 days of incubation}

ED 17 embryos were harvested by breaking open the eggs on a petridish. Blood glucose was measured using a commercially available glucometer (Accusure). The heart and liver tissues along with the amniotic fluid were collected from each embryo. The organs were weighed separately. A part of each tissue was preserved in $10 \% \mathrm{v} / \mathrm{v}$ formalin and the rest was processed for biochemical assessment.

\section{Biochemical Tests}

All the biochemical tests were performed using Mindray BA-88A Semi Auto Chemical Analyzer.

Amniotic fluid samples: The amniotic fluid samples collected from ED 17 chick embryos were centrifuged at $2000 \mathrm{rpm}$ for $20 \mathrm{~min}$. and the separated supernatants were subjected to biochemical tests to estimate total cholesterol (TC), triglycerides (TG), high density lipoprotein (HDL), using commercially available assay kits (Coral Clinical Systems, Goa, India).

Organ tissue samples: ED 17 chick embryos were sacrificed by decapitation and the hearts and livers were removed, washed with ice cold saline and weighed. A $10 \%$ homogenate was prepared in PBS, pH 7.4 and centrifuged at $10,000 \mathrm{rpm}$ for 20 mins at $4^{\circ} \mathrm{C}$. The supernatants were subjected to biochemical tests as stated above. 


\section{In vivo model ${ }^{17}$}

Male wistar albino rats weighing 250 to $300 \mathrm{~g}$ were used from CPCSEA approved Institutional Animal house of Birla Institute of Technology, Mesra, Ranchi, India vide protocol approval no. for IAEC 1972/PH/BIT/17/ IAEC. Animals were housed in polyacrylic cages and maintained under standard conditions $\left(22-25^{\circ} \mathrm{C}\right.$ and R.H. 60-65\% with 12: 12 light: dark cycles. Standard laboratory diet and water were provided.

The rats were divided into 4 groups of 6 animals each Group 1: Normal Control, fed with normal diet and injected with citrate buffer, $\mathrm{pH} 4.5$ after 4 weeks.

Remaining rats were fed with high fat diet (HFD: Lard $12 \%$, Sucrose $18 \%$, Normal diet $70 \%$ ) for 4 weeks and thereafter injected i.p with low dose streptozotocin ( $35 \mathrm{mg} / \mathrm{kg}$ body weight, in citrate buffer, $\mathrm{pH} 4.5$ ) on the $15^{\text {th }}$ day. Animals with fasting blood glucose $(\mathrm{FBG}) \geq$ $200 \mathrm{mg} / \mathrm{dL}$ after $72 \mathrm{hrs}$ of STZ injection were selected and further grouped as per the treatments which were administered 4 weeks after induction of diabetes:

Group 2: Diabetic control, HFD + STZ (35 mg/kg b.w) Group 3: HFD + STZ (35 mg/kg b.w) + Metformin (100 mg/kg b.w)

Group 4: HFD + STZ (35mg/kg b.w) + Quercetin $(100 \mathrm{mg} / \mathrm{kg}$ b.w)

HFD was withdrawn for all groups at the start of treatment which was continued for 4 weeks.

\section{Biochemical estimation of blood parameters}

Fasting blood glucose was checked at regular intervals. At the end of treatment blood was collected by retro orbital puncture under ketamine-xylazine anaesthesia and subjected to biochemical tests as done for in ovo model. Additionally $\mathrm{HbA1c}$, serum $\mathrm{LDH}$ and serum CK-MB were assessed using commercially available assay kits (Coral Clinical Systems, Goa, India). Histopathological assessment was done for heart tissues.

\section{Statistical analysis}

Statistical analysis was performed using ANOVA followed by Dunnett's test using Graph pad prism: Statistical software (trial version)

\section{RESULTS}

\section{In ovo model}

Blood glucose levels of chick embryos were checked on ED 17. Those chick embryos showing blood glucose level above $200 \mathrm{mg} / \mathrm{dl}$ were considered diabetic. This has been represented in Figure 1 where it was found that blood glucose was significantly higher in diabetic embryos as compared to normal ones $(p<0.001)$. The same was also found to be lower in metformin $(p<0.001)$ and quercetin $(p<0.01)$ treated embryos and the results were significant as compared to diabetic group.

Lipid profile of the heart, liver and amniotic fluid were tested and depicted in Figure 2. The heart tissues show a significant increase in the total cholesterol $(p<0.05)$ and triglycerides $(p<0.01)$ combined with a significant decrease in HDL $(p<0.001)$ levels in the diabetic group. TG and HDL levels were significantly reverted towards normal values in the quercetin group $(p<0.001$ for TG, $\mathrm{p}<0.01$ for HDL) and results were comparable to the metformin treated group ( $p<0.001$ for TG, $\mathrm{p}<0.05$ for HDL). Decrease in TC was significant and more evident in the quercetin treated group $(p<0.01)$ than in the metformin treated group when both were compared to the diabetic group.

In livers increased $\mathrm{TC}$ and $\mathrm{TG}$ showed significant decrease in both quercetin treated $(\mathrm{p}<0.001$ for TC, $\mathrm{p}<0.001$ for TG) and metformin treated ( $p<0.001$ for TC, $\mathrm{p}<0.01$ for TG) groups while the increase in HDL after metformin and quercetin treatments was nonsignificant when compared to diabetic group, as illustrated in Figure 3.

Amniotic fluid samples from the different groups followed the same trend in lipid profile as in the tissues. Increase in TC and TG were significantly prevented by metformin ( $p<0.01$ for TC, $p<0.001$ for TG) and quercetin treatment $(\mathrm{p}<0.05$ for $\mathrm{TC}, \mathrm{p}<0.01$ for $\mathrm{TG})$ along with a non significant increase in HDL when compared to diabetic group, as shown in Figure 4. Thus the chick embryo model confirmed the classical features of diabetes viz. hyperglycemia and hyperlipidemia.

\section{In vivo model}

Biochemical estimation of blood parameters was performed on the HFD-low dose STZ Wistar rats. Fasting blood glucose (FBG) was estimated in all animals at regular intervals. Throughout the study period, the diabetic group animals showed persistently increased FBG levels. As depicted in Figure 5. Both Metformin and Quercetin treatments significantly $(\mathrm{p}<0.001)$ brought down the FBG as compared to diabetic levels. Additionally a significant decrease in percentage $\mathrm{HbA} 1 \mathrm{c}$ in blood was found in the quercetin treated group $(\mathrm{p}<0.01)$ as well as in the metformin treated group $(\mathrm{p}<0.001)$.

Lipid profiling showed significant increase $(\mathrm{p}<0.001)$ in the levels of TC and TG. Near normal levels of TC and TG were achieved by both treatments and the effect of quercetin was comparable to metformin. Results were 


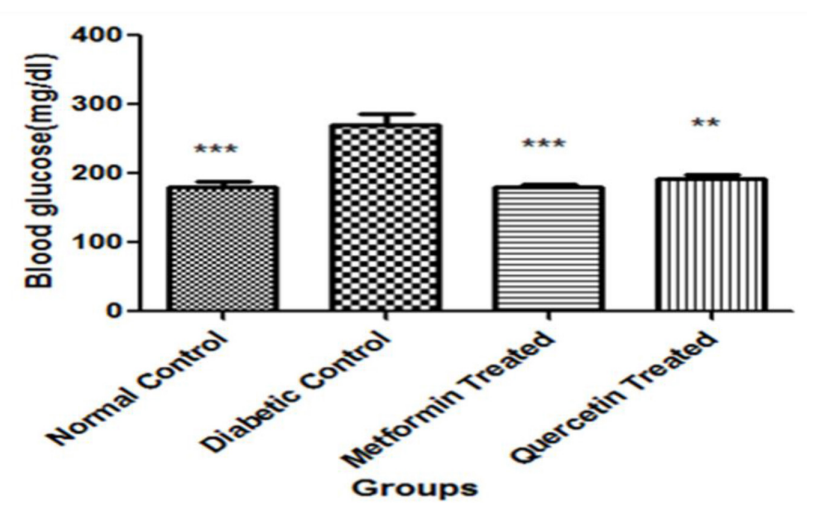

Figure 1: Blood glucose levels in ED 17 chick embryos. All values were represented as the mean \pm SEM for $n=6$. ** and ${ }^{* * *}$ indicate $p<0.01, p<0.001$ respectively when compared to diabetic control group.
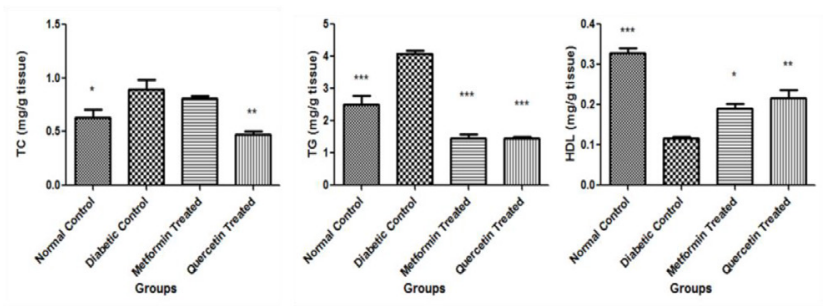

Figure 2: TC, TG and HDL levels in ED 17 chick embryo hearts.

All values were represented as the mean \pm SEM for $n=6 .{ }^{*}$, ** and ${ }^{* * *}$ indicate $p<0.05, p<0.01, p<0.001$ respectively when compared to diabetic control group.
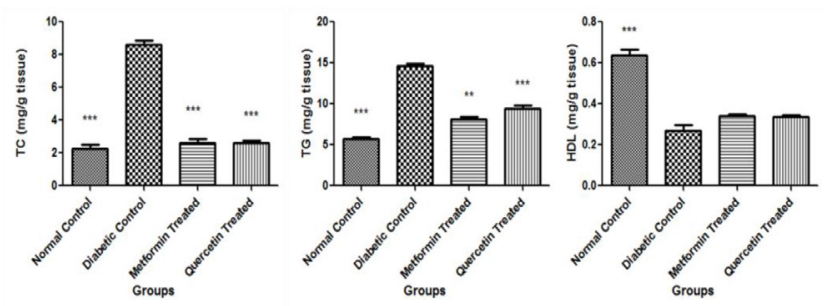

Figure 3: TC, TG and HDL levels in ED 17 chick embryo livers. All values were represented as the mean $\pm S E M$ for $n=6$. ** and ${ }^{* * *}$ indicate $p<0.01, p<0.001$ respectively when compared to diabetic control group.

significant $(\mathrm{p}<0.001$ for $\mathrm{TC}$ and $\mathrm{p}<0.01$ for $\mathrm{TG})$ when compared to the diabetic group (Figure 7). Increased atherogenic index ((total cholesterol - HDL)/HDL) in the diabetic animals was completely restored to normal by both the treatments and effects were highly significant $(\mathrm{p}<0.001)$ compared to diabetic group. The results are depicted by Figure 8.

Figure 9 shows that the level of serum LDH was found to decrease significantly $(\mathrm{p}<0.05)$ in the metformin treated group whereas quercetin treatment showed non significant decrease. Elevated CK-MB levels were

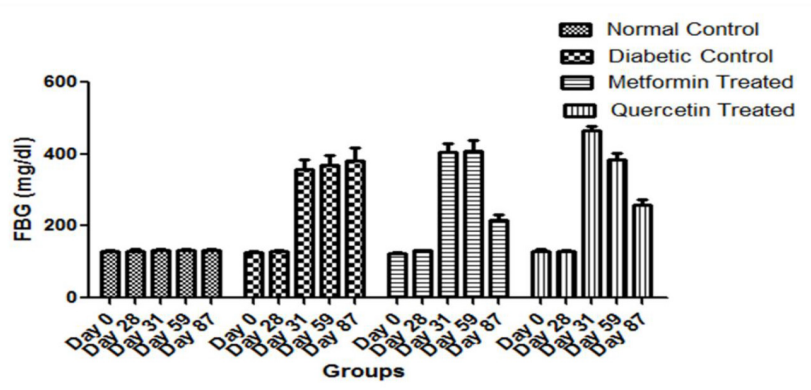

Figure 5: Variation in fasting blood glucose levels throughout study duration (0-87 days) in Wistar rats.

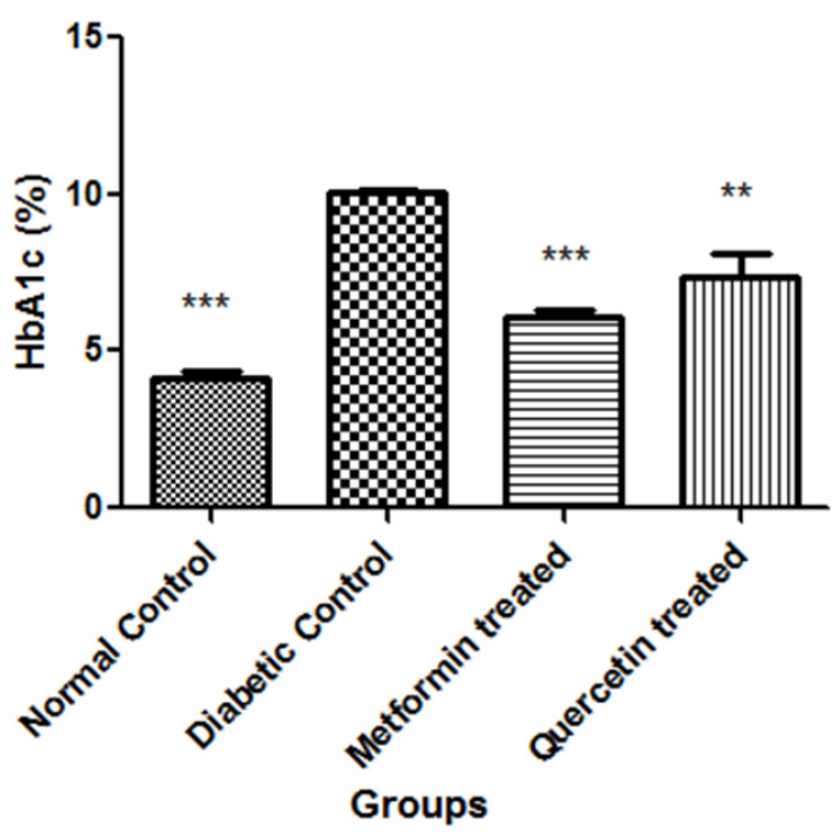

Figure 6: Percentage HbA1c in whole blood of experimental animals.

All values were represented as the mean \pm SEM for $n=6$. ** and ${ }^{* * *}$ indicate $p<0.01, p<0.001$ respectively when compared to diabetic control group. 

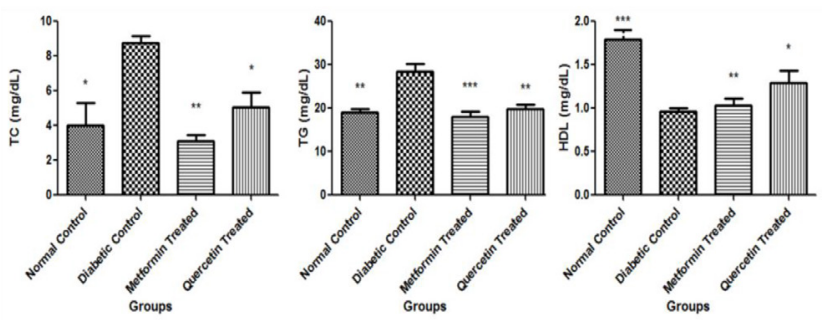

Figure 4: TC, TG and HDL levels in ED 17 chick embryo amniotic fluid.

All values were represented as the mean \pm SEM for $n=6$. ${ }^{*},{ }^{* *}$ and ${ }^{* * *}$ indicate $\mathrm{p}<0.05, \mathrm{p}<0.01, \mathrm{p}<0.001$ respectively when compared to diabetic control group.

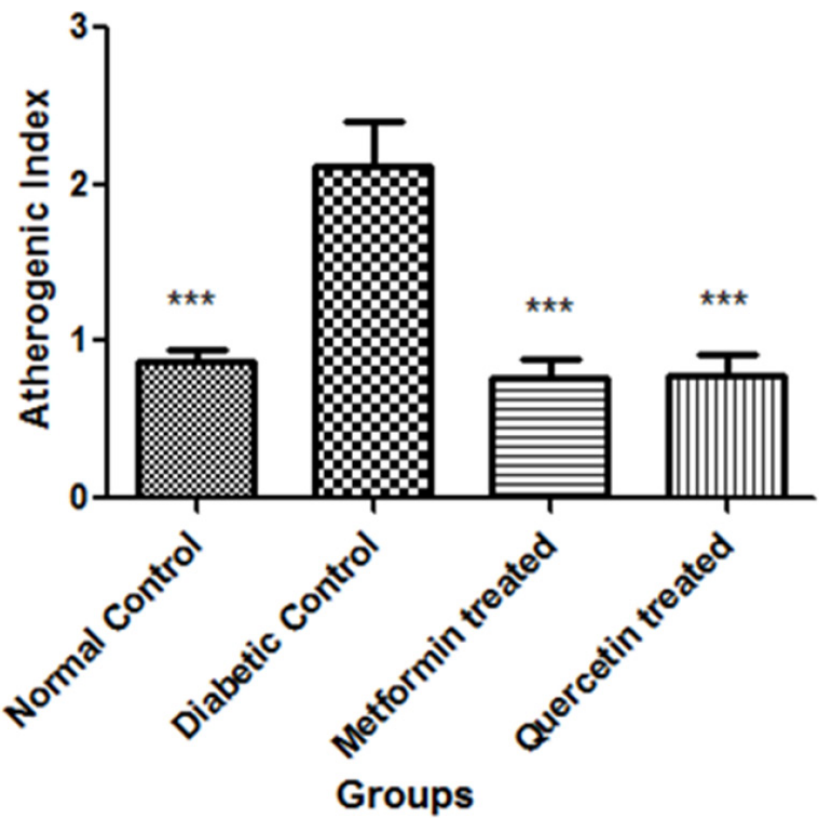

Figure 8: Atherogenic Index calculated in serum of experimental animals.

All values were represented as the mean \pm SEM for $n=6$. *** indicates $p<0.001$ when compared to diabetic control group.
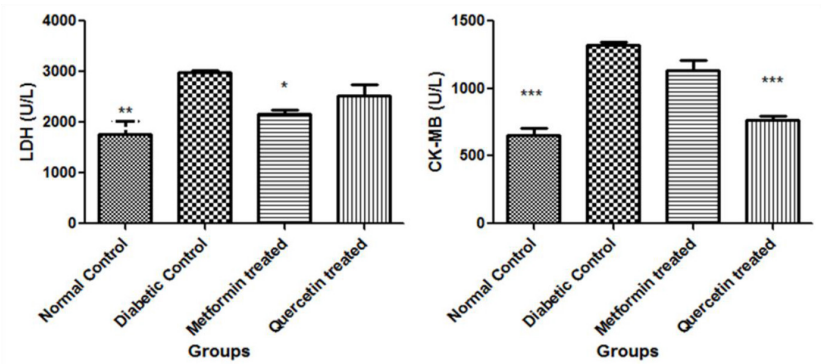

Figure 9: Serum Levels of LDH and CK-MB in experimental animals.

All values were represented as the mean \pm SEM for $n=6$. * ** and ${ }^{* * *}$ indicate $\mathrm{p}<0.05, \mathrm{p}<0.01, \mathrm{p}<0.001$ respectively when compared to diabetic control group.

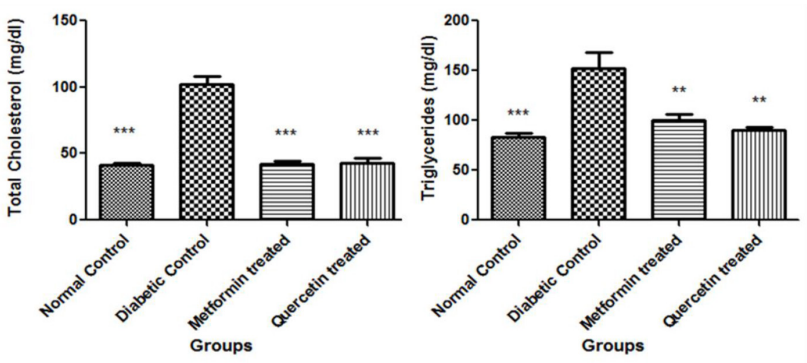

Figure 7: Plasma concentration of TC and TG in experimental animals.

All values were represented as the mean \pm SEM for $n=6$. ** and ${ }^{* * *}$ indicate $p<0.01, p<0.001$ respectively when compared to diabetic control group.
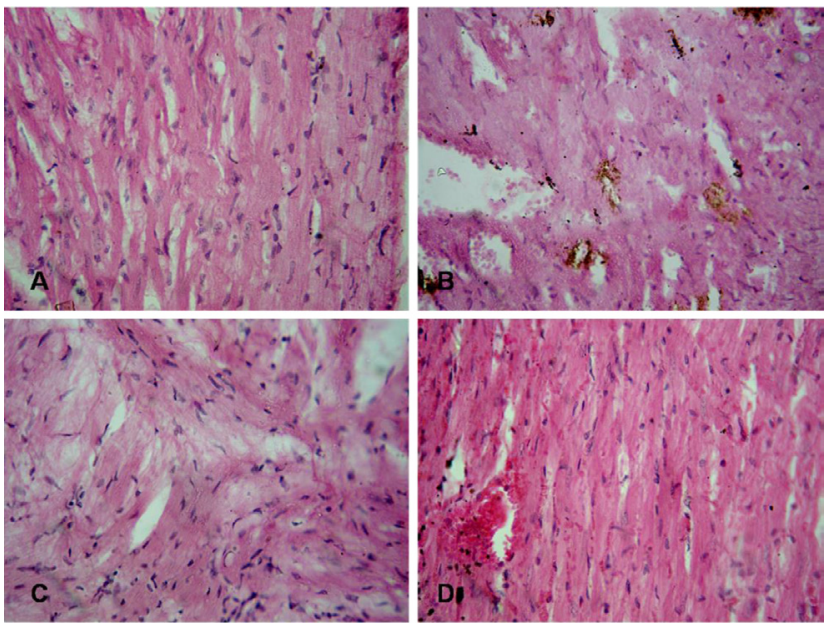

Figure 10: Histological organization of hearts of Control rats (A), Diabetic rats (B), Diabetic rats treated with Metformin (C) and Diabetic rats treated with Quercetin (D). (H and E stain, 40X).

\section{DISCUSSION}

It is hypothesized that hyperglycemia activates a number of atherogenic factors by decreasing nitric oxide, increasing oxidative stress and advanced glycation end product (AGE) synthesis and upregulation of AGE receptors. ${ }^{5}$ Earlier studies have shown that flavonoids mainly due to their antioxidant properties are useful in the treatment of diabetes mellitus. ${ }^{18}$ Quercetin was shown to preserve beta cell integrity in studies conducted by Coskun et al. ${ }^{19}$ while a review by Vizcaino et al. stated that quercetin had shown to induce a progressive, dose-dependent and sustained reduction in blood pressure in several rat models of hypertension including rat models of metabolic syndrome namely the obese Zucker rats as well as rats treated with a highsucrose, high-fat diet. It was concluded that a flavonoid 
rich diet might reduce the risk of myocardial infarction and stroke in which hypertension plays a major role. ${ }^{20}$

The work of Alam et al. showed quercetin to be effective against hyperglycaemia, oxidative stress and DNA damage in alloxan induced type 2 diabetes in rats. ${ }^{21}$ The flavonoid inhibited the early stage of glycation as evidenced by the low levels of HbA1c in quercetintreated hemoglobin samples. Quercetin also strongly inhibited the post-Amadori glycation as demonstrated by the results of HSA-glucose and HSA-MG assays proving its potential effect at almost all the stages of AGE production formation. ${ }^{22}$

The present study deals with the effect of quercetin supplement for mitigation of diabetic macrovascular complications with special focus on the cardiac aspect of this metabolic disorder. A significant decrease in the blood glucose level has been found in the chick embryos where embryos with blood glucose $>200 \mathrm{mg} / \mathrm{dl}$ have been considered diabetic. In chick, the compact myocardium with a mature trabeculae network is formed by the $9^{\text {th }}$ embryonic day and the four chambers of the avian heart are functionally equivalent to the mammalian heart. ${ }^{23}$ So the use of the chick embryo in modeling this metabolic disorder has been attempted. Atherosclerosis is said to be the major factor for coronary artery diseases. Increased cholesterol and triglyceride levels and decreased HDL levels and hyperglycemia itself are predisposing factors for atherosclerosis. ${ }^{5}$ The study shows similar trends in lipid profile in the diabetic group in chick embryos where diabetic condition was effectively simulated using glucose and $\beta$-hydroxybutyric acid (component of ketone bodies). ${ }^{16}$ Metformin and quercetin treatment effectively ameliorates these abnormalities. Quercetin treated embryos show more significant decrease $(p<0.001)$ in TC levels of heart and TG levels in liver $(p<0.001)$ than metformin treatment $(p<0.01$ in both cases) when compared to the diabetic group embryos.

The in vivo model of type 2 diabetes mellitus using HFD and low dose STZ provides more detailed insight into the role of quercetin in the said disorder. Besides causing a significant reduction in the fasting blood glucose levels, quercetin treatment has significantly decreased HbA1c levels $(p<0.01)$ and the findings are consistent with previous studies. ${ }^{24}$ According to a review on the UKPDS clinical study, risk of cardiovascular disorder in diabetes is seen to increase above a level of $6.2 \%$ and the fact that quercetin has the ability to reverse increase in $\mathrm{HbA} 1 \mathrm{c}$ rationalizes its role in the prevention of diabetic macrovascular complications if used as a dietary supplement. ${ }^{25}$ Lipid profile shows significant decrease in TC $(\mathrm{p}<0.001)$ and TG $(\mathrm{p}<0.01)$ by quercetin treatment indicating its ability to produce cardio protective effects. The probability of occurrence of atherosclerosis and subsequent prevalence of cardiovascular disease is indicated by and is directly proportional to the Atherogenic Index (A.I.). ${ }^{26}$ Flavonoid treatment is found to significantly $(\mathrm{p}<0.001)$ bring down the elevated A.I. value caused by diabetes induction proving its vasculoprotective potential. Serum lactate dehydrogenase $(\mathrm{LDH})$ and serum Creatinine kinase isozyme MB (CK-MB) are biochemical markers in clinical cardiology and their increased levels are indicative of myocardial damage. ${ }^{27}$ Diabetes caused significant increase $(p<0.01)$ in the serum level of these markers which were brought down by quercetin treatment proving its potential benefit in preventing myocardial injury.

The findings from biochemical studies were well supported by histopathological evidences. Persistant hyperglycemia in diabetes is a pertinent cause for a multitude of cells being adversely affected, among which endothelium, smooth muscle cells and platelets deserve special mention in augmenting atherogenesis and causing vascular distress. ${ }^{28}$ Histological examination of the cardiac tissues from animals of diabetic group showed extensive damage in the myocardium evidenced from necrotic and apoptotic changes. Protein degradation being an eminent effect of diabetic condition might have been responsible for structural changes in the cardiomyocytes. Oxidative stress and subsequent increase in apoptosis possibly further contributed to cellular damage and observance of features such as reduced nuclear size and disarrangement of myofibrils. ${ }^{29}$ Histopathological evidences from heart tissues of rats from various groups point towards the fact that the tissue damage caused by diabetes was effectively reversed by quercetin treatment.

\section{CONCLUSION}

Diabetes, especially type 2, is a major cause of cardiac complications. The current study has successfully generated primary evidence to prove the potential of the flavonoid, quercetin as a useful agent against diabetic macrovascular complications. Detailed and more specific biochemical tests including those for inflammatory markers, in vivo antioxidant tests, lipid peroxidation test and histopathological studies need to be performed for both chick embryo and murine models. Moreover cellular level assays for confirmation of the mechanism of action of this flavonoid are required to prove its physiological effects along with detailed histopathological evidences in support of the findings. 


\section{ACKNOWLEDGEMENT}

The authors are greatly thankful to the Department of Biomedical Instrumentation, B.I.T, Mesra, Ranchi and Faculty of Veterinary Science and Animal Husbandry, Birsa Agricultural University, Kanke, Ranchi, India, for their help and support.

\section{CONFLICT OF INTEREST}

The authors declare no conflict of interest.

\section{ABBREVIATIONS}

STZ: streptozotocin; HFD: High fat diet; HbA1c: glycated Hemoglobin; AGE: Advanced glycation end products; FBG: Fasting blood glucose; TC: Total cholesterol; TG: Triglycerides; HDL: High density lipoprotein; CK-MB: Creatinine kinase (Muscle/Brain) isoenzyme; LDH: Lactate dehydrogenase; HSA: Human serum albumin; HSA-MG: Human serum albumin-Methyl glyoxal; R.H: Relative Humidity.

\section{REFERENCES}

1. Mahgoub MA, Abd-Elfattah AS. Diabetes mellitus and cardiac function. Mol Cell Biochem. 1998;180(1-2):59-64.

2. Forbes JM, Cooper ME. Mechanisms of diabetic complications. Physiolo Rev. 2013;93(1):137-88.

3. Fowler MJ. Microvascular and macrovascular complications of diabetes. Clin Diabetes. 2008;26(2):1-10.

4. Heinonen SE, Genové G, Bengtsson E, Hübschle T, Akesson L, Hiss K, et al. Animal Models of Diabetic Macrovascular Complications: Key Players in the Development of New Therapeutic Approaches. J Diabetes Res. 2015;2015:1-14.

5. Beckman JA, Creager MA, Libby P. Diabetes and atherosclerosis: Epidemiology, pathophysiology, and management. JAMA. 2002;287(19):2570-81.

6. Traub O, Bibber RV. Role of nitric oxide in insulin-dependent diabetes mellitus-related vascular complications. West J Med. 1995;162(5):439-45.

7. Xia X, Zhang C, Liu R, Wang Z, Tang N, Liu F, et al. Effects of 20-hydroxyecdysone on improving memory deficits in streptozotocin-induced type1 diabetes mellitus in rat. Eur Pharmacol. 2014;740:45-52.

8. Srinivasan K, Viswanad B, Asrat L, Kaul CL, Ramarao P. Combination of high-fat diet-fed and low-dose streptozotocin-treated rat: A model for type 2 diabetes and pharmacological screening. Pharmacol Res. 2005;52(4):313-20.

9. Wang Z, Yuan S, Li Y, Zang Z, Xiao W, Tang D, et al. Regulation on SIRT1PGC-1a/Nrf2 pathway together with selective inhibition of aldose reductase makes compound hr5F a potential agent for the treatment of diabetic complications. Biochem Pharmacol. 2018;150:54-63.
10. King A, Bowe J. Animal models for diabetes: Understanding the pathogenesis and finding new treatments. Biochem Pharmacol. 2016;99:1-10.

11. Russell WM, Burch RL. The principles of humane experimental technique. Methuen and Co, London. 1959:69-154.

12. Shi L, Ko ML, Huang CC, Park SY, Hong MP, Wu C, et al. Chicken embryos as a potential new model for early onset type I diabetes. J Diabetes Res. 2014:1-10

13. Jiang $\mathrm{W}$, Wei $\mathrm{H}, \mathrm{He} \mathrm{B}$. Dietary flavonoid intake and the risk of coronaryheart disease: A dose dependent meta-analysis of 15 prospective studies. Throm Res. 2015;135(3):459-63.

14. Salvamani S, Gunasekaran B, Shaharuddin NA, Ahmad SA, Shukor MY. Antiartherosclerotic effects of plant flavonoids. BioMed Res Int. 2014;2014:1-11.

15. Maalik A, Khan FA, Mumtaz A, Mehmood A, Azhar S, Atif M, et al. Pharmacological application of quercetin and its derivatives: A short review. Trop J Pharm Res. 2014;13(9):1561-6.

16. Memon S, Pratten MK. Teratogenic effects of diabetic conditions in chick heart in ovo and in micromass culture may be prevented by addition of vitamin C and folic acid. Reprod Toxicol. 2013;35:117-24.

17. Jiao $Y$, Wang $X$, Jiang $X$, Kong F, Wang S, Yan C. Antidaibetic effects of Morus alba fruit polysaccharides on high-fat diet and streptozotocin-induced type 2 diabetes in rats. J Ethnopharmacol. 2017;199:119-27.

18. Nicolle E, Souard F, Faure P, Boumendjel A. Flavonoids as promising lead compounds in type 2 diabetes mellitus: Molecules of interest and structureactivity relationship. Curr Med Chem. 2011;18(17):2661-72.

19. Coskun O, Kanter M, Korkmaz A, Oter S. Quercetin, a flavonoid antioxidant, prevents and protects streptozotocin-induced oxidative stress and beta-cell damage in rat pancreas. Pharmacol Res. 2005;51(2):117-23.

20. Vizcaino FP, Duarte J, Jimenez R, Buelga CS, Osuna A. Antihypertensive effects of the flavonoid quercetin. Pharmacol Rep. 2009;61(1):67-75.

21. Alam MM, Meerza D, Naseem I. Protective effect of quercetin on hyperglycemia, oxidative stress and DNA damage in alloxan induced type 2 diabetic mice. Life Sci. 2014;109(1):8-14.

22. Alam M, Ahmad I, Naseem I. Inhibitory effect of quercetin in the formation of advance glycation end products of human serum albumin: An in vitro and molecular interaction study. Int J Biol Macromol. 2015;79:336-43.

23. Wittig GW, Munsterburg A. The early stages of heart development: Insights from chicken embryos. J Cardiovas Dev and Dis. 2016;3(2):2-17.

24. Bae JW, Lee MH. Effective and putative mechanism of action of ginseng on the formation of glycated hemoglobin in vitro. J Ethnopharmacol. 2004;91(1):137-140.

25. Turner RC. The UK. Prospective Diabetes study: A review. Diabetes Care 1998;21(3):C35-8.

26. Yang RL, Shi YH, Hao G, Le GW. Increasing oxidative stress with progressive hyperlipidemia in human relation between malondialdehyde and atherogenic index. J Clin Biochem and Nutr. 2008;43(3):154-8.

27. Pasupathi BP, Rao YY, Farook J, Bakthavathsalam G. Biochemical cardiac markers in clinical cardiology. J Medicine. 2009;10(2):100-8.

28. Othman Al, El-Sawi MR, El-Missiry MA, Abukhalil MH. Epigallocatechin-3gallate protects against diabetic cardiomyopathy through modulating the cardiometabolic risk factors, oxidative stress, inflammation, cell death and fibrosis in streptozotocin-nicotinamide -induced diabetic rats. Biomed and Pharmacother. 2017;94:362-73.

29. Cosyns B, Droomans S, Weytjens C, Lahoutte T, Van CG, Schoors D, et al. Effect of streptozotocin induced diabetes on left ventricular functions in adult rat: An in vivo pinhole gated SPECT study. Cardiovas Diabetol. 2007;6(1):30-7.

\section{About Authors}

Joyani Das: Obtained her post graduate degree from Department of Pharmaceutical Sciences \& Technology, Birla Institute of Technology, Mesra in 2015. Currently working as research scholar in the field of Pharmacology in the same department.

Dr. (Mrs.) Papiya Mitra Mazumder: Is a Professor in the Department of Pharmaceutical Sciences \& Technology, Birla Institute of Technology, Mesra. She is working on areas concerning diabetes, toxicology and effect of bioactive molecules of natural and synthetic origins. 


\section{PICTORIAL ABSTRACT}

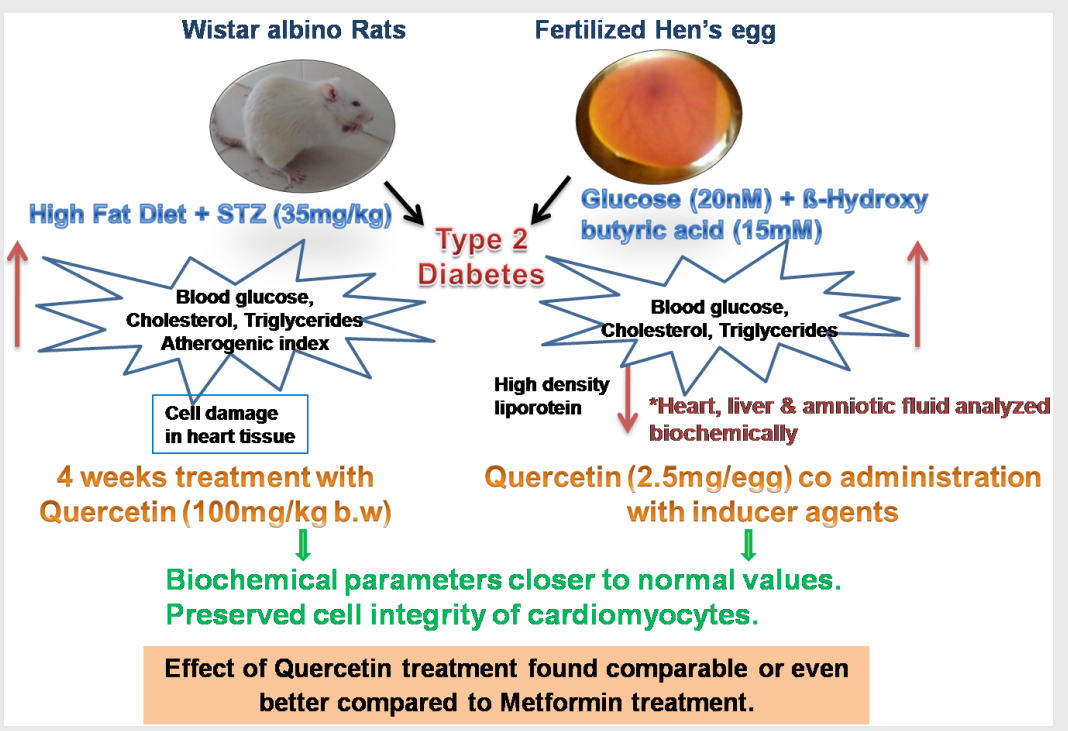

\section{SUMMARY}

- Diabetes mellitus is such a metabolic disorder which adversely affects almost all organ systems and its macrovascular complications include cardiovascular, cerebrovascular and peripheral vascular components. Flavonoids have proven health benefits due to their antioxidant, anti-inflammatory, antihypertensive, antiatherosclerotic and various other properties. The extents to which they will be effective in providing vasculoprotection in diabetic patients provided a valid target for research. Quercetin is one of the earliest known flavonoids and was hence chosen for this work.

- Wistar albino rats were fed with high fat diet (normal diet supplemented with sugar and lard) and then injected i.p with low dose streptozotocin $(35 \mathrm{mg} / \mathrm{kg}$ b.w) to induce diabetes with type 2 features which produced hyperglycemia and hyperlipidemia. These were effectively controlled after 4 weeks treatment with quercetin $(100 \mathrm{mg} / \mathrm{kg})$. Reduced atherogenic index in treated diabetic animals compared to diabetic controls proved antiatherogenic potential of the flavonoid. Increased CK-MB and LDH levels were also reversed indicating effective cardioprotection in addition to diabetes management.

- Histopathological examinations of heart tissue supported the results obtained from biochemical tests where quercetin treatment was found to prevent cellular damage to cardiomyocytes.

- Experiments have been performed in search of a non mammalian model for diabetic cardiac complications also, in which diabetes was simulated in ovo using glucose and B-hydroxybutyric acid. Quercetin, when co administered with the inducing agents, proved significantly effective in preventing elevation of blood glucose, tissue and amniotic fluid TC, TG levels and decrease in tissue and amniotic fluid HDL levels.

- In all cases metformin was used as standard drug. Quercetin was found to perform equally and in many cases better than metformin against diabetic macrovascular complications providing primary evidence of it being a potential candidate for prevention of the condition under discussion.

Cite this article: Das J, Mazumder PM. Quercetin as a Modulator of Diabetic Macrovascular Complications in Murine and Chick Embryo Models. Indian J of Pharmaceutical Education and Research. 2018;52(4):594-601. 